\title{
O ALCANCE DA EXCELÊNCIA POR PROGRAMAS BRASILEIROS DE PÓS- GRADUAÇÃO STRICTO SENSU COM DOUTORADO EM ENFERMAGEM
}

\author{
Alacoque Lorenzini Erdmann ${ }^{1}$, Josicélia Dumêt Fernandes², Valéria Lerch Lunardi³, Maria Lúcia do Carmo \\ Cruz Robazzi ${ }^{4}$, Rosalina Aparecida Partezani Rodrigues ${ }^{5}$
}
${ }^{1}$ Doutora em Filosofia da Enfermagem. Professora Titular do Departamento de Enfermagem da Universidade Federal de Santa Catarina. Bolsista 1A do CNPq. Santa Catarina, Brasil. E-mail: alacoque@newsite.com.br
${ }^{2}$ Doutora em Enfermagem. Professora Titular da Escola de Enfermagem da Universidade Federal da Bahia. Bolsista 1C do CNPq. Bahia, Brasil. E-mail: dumet@ufba.br
${ }^{3}$ Doutora em Enfermagem. Professora Associado da Escola de Enfermagem da Universidade Federal do Rio Grande. Bolsista 1 A do CNPq. Rio Grande do Sul, Brasil. E-mail: vlunardi@terra.com.br
${ }^{4}$ Doutora em Enfermagem. Professora Titular da Escola de Enfermagem de Ribeirão Preto da Universidade de São Paulo, Centro Colaborador da OMS para o desenvolvimento da pesquisa em Enfermagem. Bolsista 1B do CNPq. São Paulo, Brasil. E-mail: avrmlccr@eerp.usp.br
${ }^{5}$ Doutora em Enfermagem. Professora Titular da Escola de Enfermagem de Ribeirão Preto da Universidade de São Paulo, Centro Colaborador da OMS para o desenvolvimento da pesquisa em Enfermagem. Bolsista 1A do CNPq. São Paulo, Brasil. E-mail: rosalina@eerp.usp.br

RESUMO: Objetivou-se identificar como os programas de pós-graduação em enfermagem stricto sensu, avaliados pela Área de Enfermagem da Coordenação de Aperfeiçoamento de Pessoal de Nível Superior, no triênio 2007-2009, alcançaram a excelência - conceito 6. Estudo descritivo-exploratório, de cunho documental, que investigou os dados dos Cadernos de Indicadores destes programas, disponibilizados no site da mencionada Coordenação, em 2010. Foram analisadas 99 fichas, de 33 programas, referentes ao triênio 2007 a 2009, agrupadas nos sub-itens do quesito Inserção Social e categorias para notas 6/7. Apresentam-se os indicadores de excelência de programas norte-americanos e brasileiros, os resultados da avaliação trienal referentes aos sub-itens do quesito 5 e a descrição do desempenho dos programas indicados para o conceito 6. Conclui-se que a excelência dos programas vem sendo gradativamente conquistada, com desempenho em determinadas categorias, semelhantes às exigidas pelos padrões internacionais, vencendo-se os obstáculos para o alcance desta excelência, em sua plenitude.

DESCRITORES: Enfermagem. Educação de pós-graduação em enfermagem. Educação em enfermagem.

\section{THE ACHIEVEMENT OF EXCELLENCE IN BRAZILIAN PROGRAMS OF POST-GRADUATION STRICTO SENSU WITH A DOCTORATE IN NURSING}

\begin{abstract}
This study aimed to identify how the graduate programs in nursing stricto sensu evaluated by the Nursing field at Coordination of Improvement of Higher Education Personnel, in the triennium 2007-2009, achieved excellence - grade 6. Descriptive exploratory study of nature documentary, which examined the data of Reports Indicators of these Programs at the site of the Coordination cited, in 2010. We analyzed 99 chips of 33 programs for the triennium 2007 to 2009, grouped in sub-clauses of Social and item categories for grades 6/7. Presents the indicators of excellence of Americans and Brazilians Programs, the results of the triennial assessment relating to sub-items of item 5 and the description of program performance specified for the grade 6 . It follows that the excellence of Programs has been gradually achieved, with performance in certain categories as those required by international standards, overcoming the obstacles to the achievement of excellence in all its fullness.
\end{abstract}

DESCRIPTORS: Nursing. Education, nursing, graduate. Education in nursing.

\section{EL LOGRO DE LA EXCELENCIA EN LOS PROGRAMAS BRASILEÑOS DE POST-GRADUACIÓN STRICTO SENSU CON DOCTORADO EN ENFERMERÍA}

RESUMÉN: Este tuve como objetivo identificar los programas de postgrado en enfermería stricto sensu evaluados por el campo de la enfermería de la Coordinación de Perfeccionamiento de Personal de Nivel Superior, en el trienio 2007-2009, la excelencia alcanzado $-6^{\circ}$ grado. Estudio descriptivo exploratorio de naturaleza documental que examinó los datos de los informes de los indicadores de estos programas en el sitio de la Coordenación mencionada, en 2010. Se analizaron 99 fichas de 33 programas para el trienio 2007 a 2009, agrupadas en el sub-cláusulas sociales y categorías de artículos para los grados 6/7. Presenta los indicadores de excelencia de programas estadounidenses y brasileños, los resultados de la evaluación trienal sobre los subtemas del tema 5 y la descripción de la ejecución de los programas especificados para el grado 6. De ello se deduce que la excelencia de los programas ha ido ganando de forma progresiva, con el rendimiento en ciertas categorías como los exigidos por las normas internacionales, la superación de los obstáculos a la consecución de la excelencia en toda su plenitud.

DESCRIPTORES: Enfermería. Educación del postgrado en enfermería. Educación en enfermería. 


\section{INTRODUÇÃO}

A pós-graduação stricto sensu define o sistema de ensino após a graduação, com objetivos mais amplos e aprofundados de formação científica de docentes e pesquisadores acadêmicos. ${ }^{1}$ No Brasil, este sistema foi instituído pela Lei de Diretrizes e Bases da Educação Nacional, em 1961, e aprovado pelo Conselho Federal de Educação em 1965.

Na Enfermagem, a pós-graduação stricto sensu teve início em 1972, com a criação do curso de Mestrado da Escola de Enfermagem Anna Nery; nesta mesma década foram implantados mais sete cursos de mestrado acadêmico: quatro no sudeste, dois no nordeste e um na região sul. ${ }^{2}$ Em 1982, iniciou-se o primeiro programa de doutorado da América Latina, na área de enfermagem, desenvolvido em parceria entre a Escola de Enfermagem e a Escola de Enfermagem de Ribeirão Preto, ambas da Universidade de São Paulo.

O advento dos cursos de pós-graduação contribuiu para a constituição do habitus científico da enfermagem brasileira, mediante a realização de uma atividade de pesquisa como requisito necessário à obtenção da titulação requerida, caracterizando, assim, o estreito vínculo entre a pós-graduação e o desenvolvimento da pesquisa. ${ }^{2}$ É de vital importância a compreensão da necessidade da pós-graduação, para formar mestres e doutores em enfermagem, altamente qualificados e produtivos, em quantidade que possa compor os quadros de corpo docente, qualificando o ensino nos cursos de graduação existentes e a prática de enfermagem no país.

Os cursos de pós-graduação são avaliados sistematicamente pela Coordenação de Aperfeiçoamento de Pessoal de Nível Superior (CAPES) do Ministério da Educação, que convoca uma comissão de expertises de cada Área de conhecimento, responsável pela atribuição de conceitos de 1 a 7 , o que tem propiciado um importante elemento qualificador aos programas.

Na última avaliação trienal, referente ao triênio 2007-2009, o país contava com 41 Programas de Pós-graduação em Enfermagem (PPGEnfs) stricto sensu. Destes, 92,68 \% eram de Mestrado Acadêmico (MA) e/ou Doutorado Acadêmico (DA), e apenas três $(7,31 \%)$, de Mestrado Profissional (MP). ${ }^{3}$ A Área de Enfermagem da CAPES finalizou o ano de 2010 com um total de 48 PPGEnfs, totalizando 72 cursos, assim distribuídos: $42 \mathrm{MA}$, 23 DA e 7 MP. Destes 48 programas, 21 estavam classificados com nota 3, 14 com nota 4 , nove com nota 5 e 4 com nota $6 .{ }^{4}$
O processo avaliativo, construído com a participação da comunidade científica, é conduzido por seus pares. A enfermagem tem critérios semelhantes de avaliação de outras áreas, o que favorece a sua visibilidade e sua natureza científica. Esse modelo de avaliação tem levado os PPGEnf do Brasil, a consolidarem seus cursos. ${ }^{5} \mathrm{~A}$ avaliação é uma importante oportunidade de favorecer o amadurecimento dos programas, por propiciar uma reflexão interna sobre sua responsabilidade social, política e tecnológica, incentivando a proposição e metas para a formação de recursos humanos com excelência, nos níveis de mestrado e doutorado. ${ }^{6} \mathrm{O}$ avanço da pós-graduação em Enfermagem no país vem sendo marcado pela redução das desigualdades regionais na distribuição dos cursos e pelo incremento na sua qualificação, alcançando alguns o conceito 6, o maior até então obtido pelos PPGEnfs. Assim, este estudo objetivou identificar como os PPGEnf stricto sensu, avaliados pela CAPES e respectiva Área de Enfermagem no triênio 2007/2009, alcançaram o conceito 6, considerado de excelência.

\section{REVISÃO DA LITERATURA}

Foram apresentados aos PPGEnfs, pela Área de Enfermagem da CAPES, as diretrizes para o alcance da excelência, construídas e espelhadas em experiências nacionais e internacionais, visando fortalecer a pós-graduação da enfermagem brasileira.

\section{Indicadores de excelência internacionais para programas de doutorado em enfermagem}

Nos Estados Unidos há mais de uma modalidade de doutorado, tendo como foco a formação de pesquisadores para a produção de investigações, com o objetivo de estender o conhecimento: o Doutorado com grau de Filosofia (Doctor of Philosophy Degree - $P h D)$, direcionado à pesquisa; o Doutorado Profissional; e o Doutorado em Enfermagem (Doctor of Nursing Science - DNS)), focado na produção de conhecimentos da prática. A Associação Americana de Faculdades de Enfermagem (AACN) sugeriu indicadores de qualidade para os programas de doutorado em enfermagem ${ }^{7}$, quais sejam:

1) Professores: devem representar uma diversidade de origens e perspectivas intelectuais, apresentar dedicação, recomendando-se que assessorem não mais que três a cinco alunos durante a fase da tese. Devem ter capacidade de captar subvenção de apoio às investigações e bolsas de estudo; identificar, gerar e utilizar os recursos dentro do ambiente universitário e da comunidade 
em geral para apoiar os objetivos do programa; orientar a realização de peer-reviewed de pesquisas, teorias, ou ensaios filosóficos; criar um ambiente no qual possa haver a socialização de estudantes, bem como a existência de uma comunidade de estudiosos; ajudar os alunos a compreender o valor de programas de investigação e bolsas, entre outros. ${ }^{7}$ No Brasil, os docentes de pós-graduação devem ter ciência de seu compromisso e responsabilidade social na formação de mestres e doutores, difundindo conhecimento e propondo tecnologias e inovações resultantes de pesquisas. ${ }^{6}$ A limitação em relação ao número de alunos por professor é feita no âmbito das próprias universidades e de recomendações da Área de Enfermagem da CAPES.

2) Programa de estudo: deve ser consistente com a missão da instituição, a disciplina de Enfermagem e o grau almejado; e os requisitos e sequência de progressão devem ser claros e disponibilizados aos estudantes. O conteúdo do curso deve abordar: fundamentos filosóficos e históricos para o desenvolvimento do conhecimento em Enfermagem; métodos e processos de teoria/ desenvolvimento de conhecimento; métodos de investigação e financiamentos para a investigação, além de competências para atuação na academia, na pesquisa, na prática ou em ambientes políti$\cos ^{7}$ A Área de Enfermagem da CAPES constatou que a formação de recursos humanos nos níveis de Mestrado Acadêmico, Mestrado Profissional, Doutorado e Estágios Pós-Doutorais, no Brasil, vêm cada vez mais alcançando ótimos patamares, respeitando-se a diversidade e o livre debate de ideias, em prol de uma sociedade com melhor qualidade de vida e saúde e pleno exercício da cidadania. ${ }^{8}$ No programa de estudo podem ser inseridos elementos de ensino formal e informal, incluindo-se a imersão em experiências que auxiliarão o estudante no desenvolvimento da liderança, da prática como educador e cientista, além da socialização de oportunidades em atividades complementares aos objetivos de sua carreira. Indicadores de resultados devem incluir avaliação satisfatória do aluno nos conhecimentos adquiridos no programa; as teses devem representar contribuições originais para o campo de estudo e a avaliação sistemática do pós-graduado deve ser realizada a intervalos regulares. De 3-5 anos após a conclusão do doutorado, o egresso deve obter financiamento para sua pesquisa e deve ter utilizado o processo de investigação para resolver algum problema de importância para a enfermagem ou de cuidados de saúde no seu ambiente laboral. Empregadores devem manifestar satisfação com a liderança dos diplomados e o reconhecimento de sua liderança e conhecimento é feito diante do recebimento de prêmios, honrarias, ou financiamentos externos. ${ }^{7}$

3) Recursos: devem ser suficientes, tanto humanos como financeiros e institucionais, disponíveis para cumprir os objetivos, tanto do curso de doutorado quanto das atividades de pesquisa. A investigação deve ser considerada um componente explícito da missão institucional, deve haver um escritório para a administração de pesquisa, registros de financiamentos externos, programas de pós-doutoramentos, fundos internos para pesquisas e o ambiente universitário deve promover a colaboração e a investigação interdisciplinar. ${ }^{7}$ Em algumas universidades brasileiras esta situação acontece e os financiamentos externos de pesquisas obtidos por docentes dos programas são administrados por centros de pesquisas; a presença de enfermeiros em estágios pós-doutorais é cada vez mais incrementada.

4) Estudantes: devem ser selecionados a partir de um conjunto de candidatos altamente qualificados e motivados. Os objetivos das suas investigações devem ser congruentes com a experiência do corpo docente, de bolsa de estudos e recursos institucionais. Devem ter sucesso na obtenção de apoio financeiro e de prêmios por suas pesquisas. Parcela significativa do seu tempo deve ser dedicada ao programa, finalizando-o em tempo hábil; suas publicações e trabalhos acadêmicos devem prosseguir após a sua titulação. ${ }^{7}$ No Brasil, o corpo discente é considerado elemento-chave, por se constituir no produto da formação de recursos humanos de excelência e esta importância tem sido evidenciada pela formação qualificada e ampliação de participação dos alunos em coautorias da produção bibliográfica dos programas. ${ }^{9}$

5) Avaliação: deve ser realizada sistematicamente, de modo contínuo, abrangente e centrada na universidade, na missão específica e nas metas do programa, com adesão à ética e estabelecimento de normas para o processo de avaliação, como por exemplo, confidencialidade e rigor nas análises quantitativas e qualitativas. Requer, ainda, o envolvimento dos estudantes; a promoção de um feedback constante para os professores, administradores e componentes externos para promover a melhoria do programa; o fornecimento de dados gerais para determinar padrões e tendências e recomendar direções futuras, a intervalos regulares; e deve ter apoio adequado em relação aos recursos humanos, financeiros e institucionais. ${ }^{7}$ Ainda que aconteçam avaliações internas nas universidade que apresen- 
tam programas ou cursos de pós-graduação de excelência, a tarefa de coordenar a avaliação da pós-graduação em todas as áreas de conhecimento, incluindo-se a de enfermagem, é da CAPES, o que tem sido importante para os êxitos alcançados pelo sistema nacional de pós-graduação.

\section{Indicadores de excelência nacionais para os programas com doutorado em enfermagem}

O doutorado em enfermagem, no Brasil, assemelha-se ao $P h D$. Ainda que não se tenha um estudo minucioso sobre os egressos dos PPGEnfs nacionais, sabe-se que a maioria dos doutores formados ministra aulas, realiza pesquisas e orienta alunos de iniciação científica, mestrado e doutorado, assume funções administrativas e políticas nas academias, participa de instituições governamentais, possui capacidade de disseminar e estender o conhecimento, entre outras atribuições, assemelhadas às desejáveis em programas internacionais. ${ }^{7}$ Investigação realizada com indivíduos titulados em diversas áreas de conhecimento mostrou que a formação recebida na pós-graduação parece ter preenchido bem as expectativas e demandas das profissões acadêmicas. ${ }^{10}$

Programas de pós-graduação de excelência nacionais, além de atender aos demais critérios de avaliação, devem se destacar nas categorias: Solidariedade, Nucleação, Liderança e Internacionalização. ${ }^{11}$ A Solidariedade é entendida como: cooperação com outros programas com conceitos 3 ou 4 ou com grupos que ainda não apresentam curso de pós-graduação stricto sensu; atuação em rede, para diminuir os desequilíbrios regionais na oferta e desempenho da pós-graduação e atender às novas áreas de conhecimento, buscando promover e/ou consolidar cursos de pós-graduação; assessoria para a formulação de propostas de cursos novos nacionais ou internacionais; participação em projetos conjuntos com grupos de pesquisa não consolidados; participação em disciplinas, seminários e oficinas em outros cursos e programas em fase de consolidação, realização de eventos de abrangência nacional e internacional para qualificar docentes e pesquisadores da área; e parceria de docência, pesquisa e orientação em países com menor grau de desenvolvimento na pós-graduação. ${ }^{11}$ Nucleação é a capacidade para preparar estudantes críticos para tornarem-se educadores, pesquisadores e líderes no âmbito da saúde e, mais especificamente, da enfermagem. Neste aspecto é importante que os egressos tenham participação em: atividades de coordena- ção e ensino de graduação e pós-graduação e em pesquisa, em outras Instituições de Ensino Superior (IES) da região, em outras regiões do país ou em países com menor grau de desenvolvimento; atividades administrativas no setor público ou privado, em órgãos de gestão de classe e associações científicas da área; e em coordenações acadêmicas ou comissões/assessorias de abrangência nacional em políticas públicas de saúde, educação, ciência e tecnologia ${ }^{11}$, atribuições estas também encontradas em programas de excelência norte-americanos. ${ }^{7}$ A Liderança é representada pela atração de alunos de diferentes regiões do próprio país ou de outros; atração, pelo corpo docente de doutores, do país e/ou do exterior, para estágios pós-doutorais e as participações de docentes em: comitês de área de agências (CNPq, FINEP, CAPES ou de agências de fomento estaduais e internacionais); diretorias de associações científicas nacionais e internacionais; cargos relevantes para a política nacional de saúde, educação e/ou ciência e tecnologia; conferências ou palestras em eventos científicos relevantes, no país e no exterior; e formulação das políticas públicas de saúde e educação. O corpo docente deve ter apoio financeiro para desenvolvimento de pesquisas e Bolsa de Produtividade/CNPq e receber premiações nacionais e/ou internacionais, relacionadas às atividades de ensino, pesquisa e orientação ${ }^{11}$, atribuições assemelhadas às constantes para os programas norte-americanos. ${ }^{7} \mathrm{Na}$ Internacionalização do programa são avaliadas as participações internacionais, que se referem as seguintes participações: de docentes como visitantes em programas de IES estrangeiras; em treinamento de pós-doutorado em programas no exterior; de orientadores de doutores de outros países em estágio pós-doutoral; em intercâmbios e convênios de cooperação internacional caracterizados por reciprocidade entre as IES brasileiras e estrangeiras de reconhecimento internacional da área; de professores visitantes estrangeiros recebidos pelo PPGEnf no triênio; de intercâmbio de alunos com IES estrangeiras, sobretudo por meio de bolsas-sanduíche; de docentes e discentes em eventos científicos de caráter internacional; na organização de eventos acadêmico-cientificos internacionais; em comitês editoriais e em editoria de periódicos qualificados de circulação internacional; em comitês e diretorias de associações, sociedades científicas e programas internacionais; em projetos de pesquisa envolvendo programas de pós-graduação e grupos de pesquisa de IES no exterior; e na captação de recursos de agências de fomento científico de âmbito internacional. ${ }^{11}$ Os indicadores de 
produção de circulação internacional avaliados são evidenciados por: expressão científica e social no contexto internacional (publicações em periódicos e livros qualificados de circulação internacional); distribuição da produção intelectual/cientifica de forma equilibrada entre os docentes, com qualidade equivalente à de programas de destaque internacional sediados no exterior; e evidência de impacto da produção cientifica e tecnológica na Área de Enfermagem, como número de citações, impacto nacional, impacto em políticas públicas e outros específicos. ${ }^{11}$

Os programas de enfermagem também devem apresentar perfil de excelência internacional em competências/aptidões/domínios na formação de seus egressos, quais sejam: 1. domínio do estado da arte em sua temática/área de atuação, com capacidade de diálogo em âmbito internacional e compreensão em similar nível de alcance, argumentação na sustentação de suas idéias perante seus pares e em outros campos de conhecimento na comunidade científica rumo à inserção e construção de parcerias ou redes de produção de conhecimentos; 2 . domínio da especificidade da área em abrangência e profundidade, em determinada temática ou interfaces de conhecimentos com a mesma, de modo a contribuir para o seu avanço, incorporando novos saberes e fazeres e prática de cuidado interdisciplinar; 3. identificação e promoção de novos caminhos no conhecimento, visando a distinção científica e tecnológica e inserção social, para a consolidação e fortalecimento da identidade da Enfermagem; 4. percepção e interpretação das oportunidades do desenvolvimento de novos conhecimentos, avaliando sua importância para o campo teórico e prático da área, com base nos impactos dos diversos saberes; 5 . habilidades/competências para a pesquisa, coordenação de equipes e empreendimento do conhecimento com habilidades conexas na gestão de projetos de pesquisa e prospecção de oportunidades em pesquisa; 6. expert em métodos científicos e/ou criador de novos métodos e tecnologias para o processo de construção de conhecimentos avançados, bem como, domínio dos instrumentos e processo de divulgação/socialização do conhecimento em periódicos qualificados; 7. exercício do processo educativo, colaborando na formação de novos pensadores/profissionais para competências/aptidões em conhecimentos ou saberes da área da Enfermagem e/ou áreas afins, com visão crítico-reflexiva, construtiva e colaborativa; e 8. capacidade de construção de projeto de carreira científica, considerando sua liderança, inserção, reconhecimento acadêmico, além do tempo de vida profissional, interesse, vontade, necessidades ou condições pessoais. ${ }^{11}$

Considera-se, ainda, a quantidade e qualidade dos Docentes Permanentes (DP) portadores de bolsa de produtividade em pesquisa do $\mathrm{CNPq}$ e seus respectivos projetos de pesquisa cooperativos e multicêntricos, financiados por agencias nacionais e internacionais, propiciando a realização de estágios de doutorados sanduíche dos alunos e os pós-doutorados dos docentes, com titulação qualitativa e quantitativa de alunos doutores por DP, publicações qualificadas dos docentes e respectivos orientandos e o número de alunos de outros programas e países presentes nos grupos pesquisa em atividades de estágio ou doutorado sanduíche e de doutores em estágio pós-doutoral. ${ }^{11}$ Docentes da pós-graduação envolvidos nos cursos de graduação e de pós-graduação, na liderança de grupos e na condução de projetos integrados de pesquisa articulados com seus orientandos de vários níveis, lapidando talentos para a futura geração de pesquisadores. ${ }^{6}$

Programas relevantes apresentam desempenho em práticas inovadoras de formação de recursos humanos em pesquisa e produção de conhecimentos avançados na área, atuação ou formação de redes inovadoras de pesquisa e pós-graduação regionais, nacionais e internacionais, mostrando a efetiva cooperação entre pesquisadores, diminuindo os desequilíbrios regionais com a oferta de Dinter/Minter e outras modalidades, promovendo a criação e fortalecimento de linhas e grupos de pesquisas e cursos de pós-graduação e incrementando inovações tecnológicas e apropriação de conhecimentos científicos e tecnológicos, dentre outros aspectos pertinentes ao caráter técnico e político de funcionalidade de um programa destacado. Com isso viabiliza-se a ampliação de conhecimentos ou saberes da área em consonância às políticas públicas e consolidação do Sistema Único de Saúde (SUS), com sustentabilidade e empreendedorismo social com impacto significativo do cuidado de enfermagem na sociedade, ao dispor de mais saúde e melhor cuidado realizado por profissionais da enfermagem melhor qualificados. ${ }^{11}$

\section{MATERIAL E MÉTODOS}

O estudo é descritivo-exploratório, de cunho documental e abordagem quantitativa. A coleta de dados foi realizada em julho de 2010. Os documentos consultados referem-se aos Cadernos de 
Indicadores dos Programas com as modalidades de MA e DA, disponíveis na página da CAPES (www.capes.gov.br). Foi efetuada consulta às fichas denominadas Propostas dos Programas (PP), preenchidas pelos coordenadores de PPGEnf, pois nelas se encontram as informações sobre os quesitos Solidariedade, Nucleação e Visibilidade. Foram analisadas 99 fichas de 33 programas, do triênio 2007-2009. Além disso, também foram analisadas 33 Fichas de Avaliação (FA), disponibilizadas pela CAPES, nas quais constava o quesito Inserção Social, que representava 15\% da avaliação total de cada programa, e é composto pelos seguintes sub-itens: 1 . inserção e impacto regional e (ou) nacional do programa; 2. integração e cooperação com outros programas e centros de pesquisa; e 3. desenvolvimento profissional relacionados à área de conhecimento do programa, com vistas ao desenvolvimento da pesquisa e da pós-graduação. Cada sub-item foi avaliado como fraco, regular, bom e muito bom. O sub-item 1 corresponde a $30 \%$ do valor total do quesito Inserção Social e avalia a contribuição dos PPGEnf para a melhoria do ensino, geração de materiais didáticos, formação de recursos humanos para a administração pública/sociedade civil, desenvolvimento regional e/ou nacional, e disseminação de técnicas e conhecimentos. O sub-item 2 (55\% do quesito), avalia a participação dos PPGEnfS em programas de cooperação (Minter, Dinter, Projetos Temáticos, entre outros); elaboração de estratégias para a mobilidade e a cooperação de docentes e discentes entre programas de diferentes IES por meio de publicações conjuntas, parcerias em eventos, intercâmbios docentes e redes de pesquisas inter-institucionais. O sub-item 3 (15\% do quesito) avalia: a manutenção da página do programa na $W E B$ em mais de um idioma, com dados sobre: produção intelectual, normatizações do programa, teses e dissertações, processo seletivo, regimento e estrutura curricular, disciplinas oferecidas e planos de ensino, grupos de pesquisa e financiamentos recebidos.

Para a classificação dos Programas de Doutorado em Enfermagem como de excelência, estabeleceu-se como critérios de inclusão, que eles deveriam titular doutores em enfermagem, com perfil de excelência internacional em algumas competências/aptidões/domínios, conforme já descrito. ${ }^{8}$ Foram, ainda, considerados os indicadores das categorias Solidariedade, Nucleação, Liderança e Internacionalização ${ }^{11}$, além de elementos como quantidade e qualidade dos DPs portadores de bolsa de produtividade em pesquisa e seus respectivos projetos de pesquisa cooperativos e multicêntricos, financiados internacionalmente, número de alunos em estágios de doutorados-sanduíche dos alunos; quantidade de docentes em estágios de pós-doutorados; publicações qualificadas dos DPs e orientandos; presença de alunos estrangeiros; presença de práticas inovadoras de formação de recursos humanos em pesquisa e produção de conhecimentos avançados na área, atuação em rede ou formação de redes inovadoras de pesquisa e pós-graduação regionais, nacionais e internacionais; ofertas de Dinter/Minter e outras modalidades, entre outros aspectos já anteriormente descritos.

As informações foram compiladas e analisadas em conjunto, avaliando-se as informações dos coordenadores e dos avaliadores. Por se tratar de pesquisa com utilização de dados secundários, o estudo não foi submetido a Comitê de Ética em Pesquisa.

\section{RESULTADOS}

Informações de coordenadores e avaliadores coincidiram, na maioria das vezes, quanto ao quesito Inserção Social. A avaliação de todas as PPs, conforme as autoavaliações realizadas pelos próprios PPGEnfs, referentes a este quesito e seus sub-itens 1 (INS - inserção e impacto regional e (ou) nacional do programa), 2 (INT - integração e cooperação com outros programas e centros de pesquisa) e 3 (DES - desenvolvimento profissional relacionados à área de conhecimento do programa), encontra-se apresentada a seguir.

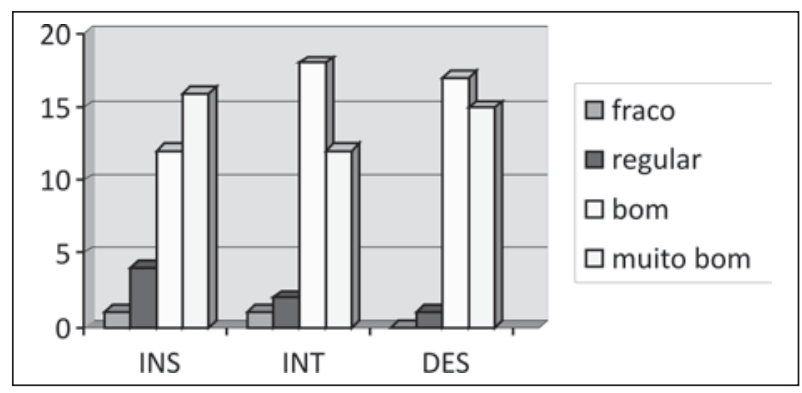

Figura 1 - Distribuição de Programas de PósGraduação em Enfermagem brasileiros, de acordo com o quesito Inserção Social e os sub-itens INS (inserção e impacto regional e(ou) nacional do programa), INT (integração e cooperação com outros programas e centros de pesquisa) e DES (desenvolvimento profissional relacionados à área de conhecimento do programa), conforme a autoavaliação contida nas Propostas dos Programas. Brasília, 2007-2009 (n=33). 
A análise realizada pela Área da Enfermagem da CAPES apontou, entretanto, que em relação aos três sub-itens, apenas um PPGEnf enquadrava-se como fraco; dois foram avaliados como regulares; 18 como bons; e 12 PPGEnfs como muito bons.

Quanto ao desempenho dos quatros programas que foram indicados para o conceito 6, nas categorias determinadas para esse conceito, ficou evidente que se enquadravam na grande maioria dos indicadores estabelecidos para notas 6 e 7 estabelecidos para a Área de Enfermagem. ${ }^{8}$ Tais programas estão localizados nos Estados de São Paulo (três deles são de IES de uma mesma universidade) e Santa Catarina e apresentaram desempenho e estrutura de referência no país, evidenciando as características de programas de excelência, compatível com os dos programas de doutorado de universidades do exterior ${ }^{7}$, conforme ficou registrado em suas FAs. ${ }^{4}$

Ainda, nos 33 PPGEnfs analisados, evidenciou-se que a autoavaliação dos coordenadores de programa e a dos avaliadores coincidiram nos três sub-itens, principalmente em relação ao conceito bom; todavia, neste conceito, as autoavaliações relativas às PPs mostraram-se mais generosas do que as FAs preenchidas pelos membros da Comissão de Avaliação da Área. Tornou-se clara a existência de um movimento nos PPGEnfs brasileiros, que busca o alcance de padrões de excelência conforme os indicativos internacionais. A excelência dos PPGEnfs resulta de políticas e estratégias para incrementar a qualificação, tendo como meta o alcance do conceito máximo sete.

\section{DISCUSSÃO}

Um fato que chama a atenção na evolução da ciência da enfermagem, no Brasil, é a distribuição assimétrica dos programas, encontrando-se concentrados, expressivamente, na região sudeste, permanecendo esparsos nas demais regiões do país. Esta situação também se assemelha com a de outros campos de conhecimentos. ${ }^{12}$ Há uma premente necessidade de expansão dos PPGEnfs para onde não existe esse nível de ensino ${ }^{2}$, e incremento à demanda para formação de novos doutores, o que pode favorecer a ampliação dos programas em regiões menos favorecidas. ${ }^{9}$

Os programas avaliados como de excelência apresentavam, conforme as recomendações da Área de Enfermagem ${ }^{11}$, no que se refere à Solidariedade: atuação em rede com vista à diminuição dos desequilíbrios regionais na oferta e desempenho da pós-graduação e promover e/ ou consolidar os cursos de pós-graduação. Os docentes assessoravam a formulação de propostas de cursos novos; participavam em projetos conjuntos com grupos de pesquisa não consolidados, especialmente em programas em fase de consolidação; promoviam eventos de abrangência nacional e internacional, para qualificar docentes e pesquisadores da Área, e realizavam parcerias de docência, pesquisa e orientação em países com menor grau de desenvolvimento na pós-graduação. Estas informações encontram-se disponibilizadas na CAPES. ${ }^{3-4}$ Quanto à Nucleação: os egressos participavam em atividades de pesquisa, coordenação e ensino de graduação e pós-graduação em outras IES da região, em outras regiões do país ou em países com menor grau de desenvolvimento, tanto na América Latina como na África; exerciam atividades administrativas no setor público ou privado, em órgãos de gestão de classe e associações científicas da área, em coordenações acadêmicas ou comissões/assessorias de abrangência nacional, em políticas públicas de saúde, educação, ciência e tecnologia ${ }^{3-4}$, a exemplo das recomendações da Área de Enfermagem ${ }^{11}$ e dos critérios de excelência internacionais. ${ }^{7}$ Ainda, quanto à Liderança: participação dos docentes em funções relevantes nos comitês de área do CNPq, FINEP, CAPES, FAPESP, ou de agências de fomento estaduais e internacionais, e em diretorias de associações científicas nacionais e internacionais; receberam premiações nacionais/internacionais, particularmente de pesquisa; proferiram conferências ou palestras em eventos científicos relevantes, no país e no exterior; obtenção de apoio financeiro para realizar pesquisas, com bolsa de produtividade em pesquisa e participação em comitês para formulação de políticas, principalmente na área de saúde e educação, atendendo, assim, as recomendações nacionais e internacionais. ${ }^{7,11}$ Apesar de, na maioria das vezes, em decorrência do próprio perfil da economia brasileira, os programas não contarem com abundância de recursos humanos, de materiais e de financiamento como os de países mais avançados, os quatro melhores avaliados pela Área de Enfermagem da CAPES apresentam a maioria dos critérios internacionais descritos. ${ }^{7}$ Referente a Internacionalização: os orientadores realizaram visitas ao exterior; atividades de pós-doutorado no país e no exterior; participaram de intercâmbios e convênios de cooperação internacional; receberam visitantes estrangeiros; direcionaram os douto- 
randos aos intercâmbios com IES estrangeiras; receberam docentes de universidades europeias, americanas, canadenses e latino-americanas para participarem de comissões julgadoras de defesas de teses de doutorado. Além disso, participaram em eventos científicos de caráter internacional, na organização de eventos científicos internacionais, foram editores dos principais periódicos da Área de Enfermagem e/ou participaram de comitês editoriais ou redigiram editoriais para periódicos de circulação internacional. Participaram em comitês e diretorias de associações, sociedades científicas e programas internacionais e em projetos de pesquisa envolvendo programas de pós-graduação e grupos de pesquisa de instituições no exterior; e captaram recursos de agências de fomento científico de âmbito internacional, conforme recomendações da Área ${ }^{11}$, e as internacionais. ${ }^{7}$

O desempenho dos quatros programas indicados para o conceito 6 correspondeu à grande maioria dos indicadores estabelecidos atribuições de notas 6 e 7, estabelecidos para a Área de Enfermagem, ${ }^{11}$ e apresentavam desempenho compatível com os dos programas de universidades do exterior ${ }^{7}$, consideradas de referência para a Área ${ }^{11}$, conforme apresentado no site da Capes. ${ }^{4}$

Considerando-se que os PPGEnfs são constituídos, essencialmente, por professores e alunos, o empenho e trabalho harmonioso destes parceiros é que vai garantir, ao final, um maior conceito atribuído pela avaliação da CAPES, e a sua possibilidade de figurar entre cursos reconhecidos como de excelência. Quanto maior o conceito recebido, maiores são as possibilidades de atração de alunos de outros países, de realização de convênios internacionais, de docentes efetuando intercâmbios, de obtenção de verbas, de projetos de pesquisas qualificados, entre outros, o que incrementaa visibilidade nacional e internacional dos programas.

Ainda em relação à Internacionalização, é fortemente recomendada a divulgação do conhecimento da pós-graduação brasileira, em periódicos internacionais, com elevado fator de impacto. Esta distribuição deve ser equilibrada, considerando-se o conjunto de professores do programa. ${ }^{11}$ Divulgar as pesquisas, por meio de publicações, é um compromisso de ordem acadêmica e social, particularmente se foram financiadas com recursos públicos. ${ }^{13}$

Constata-se, então, que a pós-graduação em enfermagem brasileira vem se fortalecendo e consolidando seus cursos e preocupando-se em manter e/ ou aumentar os seus conceitos, visando maior visibilidade. Vem crescendo tanto em número de PPGEnf stricto sensu e expansão de cursos, como na qualidade da produção de conhecimentos científicos ou tecnológicos avançados, publicados em periódicos de impacto e na formação de recursos humanos qualificados. ${ }^{14} \mathrm{~A}$ experiência acumulada por meio da produção, organização e comunicação do conhecimento da ciência da enfermagem tem se constituído em uma ação propulsora que a coloca em destaque no contexto mundial, sem nada a desejar às demais áreas. ${ }^{6}$ Sua importância no cenário nacional é notória. É um campo de conhecimento científico, tecnológico e de inovação e uma prática social relevante e compromissada com as políticas públicas de atenção à saúde, articulada ao SUS, que promove qualidade de vida por meio de um cuidado de enfermagem de excelência, caracterizando-se como uma disciplina própria com interface em diversos campos do saber. ${ }^{8}$

Entre as políticas discutidas e emanadas desta Área, para o avanço da enfermagem como ciência, tecnologia e inovação, encontram-se as propostas de incrementar a política de expansão e articulação dos programas de pós-graduação, visando a excelência da formação de mestres e doutores para o avanço da ciência, tecnologia e inovação em enfermagem e saúde e formação mais qualificada dos novos profissionais enfermeiros; promover a transferência de conhecimento da ciência de enfermagem para a sociedade, em sintonia com as políticas públicas, contribuindo para a consolidação do SUS; estimular a criação de ambientes de tecnologia e inovação para desenvolvimento de modelos de cuidado de enfermagem e saúde, com estratégias de sustentabilidade e empreendedorismo; investir na criação de indicadores de impacto econômico e social, de tecnologia e inovação em enfermagem e saúde; promover a criação de redes de desenvolvimento de inovação e tecnologia em enfermagem e saúde, para garantir um cuidado de enfermagem seguro e de excelência, entre outras. ${ }^{8}$

Estas medidas estão em consonância com as diretrizes/recomendações de busca pela excelência. Trabalho árduo, esforço coletivo, solidariedade dos programas mais fortes aos que se encontra em consolidação, mais recursos por parte dos órgãos competentes e maior empenho das universidades certamente constituem-se em etapas a serem percorridas para que os desafios da enfermagem 
brasileira sejam vencidos e os problemas existentes possam ser minimizados. ${ }^{14} \mathrm{~A}$ excelência foi alcançada ao obter o conceito 6 do seu primeiro programa, em 2007, como resultado da avaliação trienal, de 2004 a 2006, e mais outros três, em 2010, da avaliação trienal 2007-2009. Também corroboraram para isto, a conquista das indexações no Web of Science de periódicos internacionais de enfermagem editados no Brasil, a partir de 2007, chegando-se a 4 em 2010.

Em síntese, a consolidação da excelência da pós-graduação da enfermagem brasileira reflete a maturidade e solidez de desempenho alcançados pelos programas em equivalência aos existentes nos Doutorados de Enfermagem ou centros internacionais de excelência e de referência para a Área, como resultado da qualificação que se diferenciou dentre os programas que atenderam os requisitos para a nota 5, em especial, pela visibilidade e inserção no âmbito internacional, nas categorias: Solidariedade, Nucleação, Liderança e Internacionalização dos PPGEnfs.

\section{CONSIDERAÇÕES FINAIS}

Os desafios para o alcance da excelência nos programas de doutorado em enfermagem vêm sendo gradativamente vencidos, e alguns apresentam desempenho em determinadas categorias, semelhantes às exigidas pelos padrões internacionais. Obstáculos são enfrentados e vencidos frente o alcance da almejada excelência na pós-graduação de Enfermagem brasileira, marco histórico para a Área, ao ter o conceito 6 do seu primeiro programa, em 2007, pela avaliação trienal de 2004-2006, seguidos de outros três, em 2010, da Avaliação Trienal 2007-2009, e expectativa de obter na próxima avaliação trienal, o almejado conceito/nota sete.

Com o crescimento do número de programas e com a estratégia utilizada pela Área de Enfermagem na CAPES, de encontros periódicos e frutíferos com os programas, estes estão gradativamente sendo fortalecidos, garantindo assim a qualidade necessária para o avanço na consolidação da ciência, tecnologia e inovação na enfermagem através da formação de mestres e doutores em enfermagem altamente qualificados e contributivos para a sociedade. Trata-se de um esforço coletivo, agregador e integrador de potenciais para o fortalecimento, visibilidade, reconhecimento e valorização da enfermagem brasileira.

\section{REFERÊNCIAS}

1. Hueb W, Mady C, Ramires JAF. Trinta anos de pósgraduação em Cardiologia. Arq Bras Cardiol. 2005 Dez; 85(6):385-7.

2. Santos TCF, Gomes, MLB. Nexos entre pósgraduação e pesquisa em enfermagem no Brasil. Rev Bras Enferm. 2007 Jan-Fev; 60(16):91-5.

3. Coordenadoria de Aperfeiçoamento de Pessoal de Nível Superior. Relação de cursos recomendados e reconhecidos. Grande Área Ciências da Saúde, Área Enfermagem [página da internet]. [acesso 2010 Set 11]. Disponível em: http://conteudoweb.capes. gov.br/conteudoweb/ProjetoRelacaoCursosServl et?acao $=$ pesquisarIes\&codigoArea $=40400000 \&$ des cricaoArea $=\mathrm{CI} \% \mathrm{CANCIAS}+\mathrm{DA}+\mathrm{SA} \% \mathrm{DADE}+\& \mathrm{de}$ scricaoAreaConhecimento=ENFERMAGEM\&des cricaoAreaAvaliacao $=$ ENFERMAGEM

4. Coordenadoria de Aperfeiçoamento de Pessoal de Nível Superior. Resultados Avaliação Trienal. Resultados Tabela por Área [página da internet]. [acesso 2010 Out 30]. Disponível em: http:/ / trienal. capes.gov.br/wp-content/uploads/2010/09/ Resultados-por-Área.pdf

5. Rodrigues RAP, Erdmann AL, Silva IA, Fernandes JD, Araújo TL, Carneiro et al. Educação doutorado em enfermagem no Brasil. Rev Latino-am Enfermagem [online]. 2008 Ago [citado 2011 Nov 08]; 16(4):665-71. Disponível em: http:/ /www. scielo.br/scielo.php?script=sci_arttext\&pid=S0104$11692008000400003 \& \operatorname{lng}=\mathrm{en}$

6. Oliveira AC, Ferreira MA. Editorial. O papel estratégico do corpo docente no programa de pós-graduação. Esc Anna Nery. 2011: Abr-Jun, 15(2):227-9.

7. American Association of Colleges of Nursing. Indicators of Quality in Research-Focused Doctoral Programs in Nursing. [acesso 2011 Fev 02]. Disponível em: http://www.aacn.nche.edu/ publications/positions/qualityindicators.htm

8. Erdmann AL, Fernandes JD. Programas de pósgraduação em enfermagem no Brasil: desafios e perspectivas. Esc Anna Nery [online]. 2011 JanMar [acesso 2011 Nov 06]; 15(1):7. Disponível em: http:/ / www.scielo.br/scielo.php?script=sci arttext\&pid=S1414-81452011000100001\&lng=pt

9. Lima MADS, Rodrigues RCM. Editorial. O papel estratégico do corpo discente nos programas de pós-graduação. Esc Anna Nery. 2011 Jul-Set, 15(3):451-53.

10. Velloso J. Editorial. A pós-graduação no Brasil: formação e trabalho de mestres e doutores no país. Cad PesQ. 2004 Mai-Ago; 34(122):517.

11. Indicadores de sustentação dos conceitos 6 e 7 - Área de Enfermagem da CAPES - Triênio2007/2009. Documento de Área, 2010. [página da internet] 2010 [acesso 2011 Set 11]. Disponível em: http:/ / trienal. 
capes.gov.br/wp-content/uploads/2010/09/ ENFERMAGEM-rel-11set10.pdf

12. Carvalheiro JR. Janus bifronte e a pós-graduação. Ciênc Saúde Coletiva. 2010; 15(4):1908-16.

13. Coimbra Jr CEA. Efeitos colaterais do produtivismo acadêmico na pós-graduação. Cad Saúde Pública. 2010 Out; 25(10):2093.
14. Robazzi MLCC. O desempenho da pós-graduação stricto sensu em enfermagem e a procura pela excelência. Editorial. Rev Latino-am Enfermagem [online]. 2010 Nov [acesso 2010 Nov 08]; 18(5):839-40. Disponível em: http://www.scielo. br/scielo.php?script $=$ sci_arttext\&pid $=$ S0104$11692010000500001 \& \operatorname{lng}=$ en\&nrm=iso
Correspondência: Maria Lúcia do Carmo Cruz Robazzi Universidade de São Paulo, Escola de Enfermagem de Ribeirão Preto

Departamento de Enfermagem Geral e Especializada

Avenida dos Bandeirantes, 3900

14040-902 - Ribeirão Preto, SP, Brasil

E-mail: avrmlccr@eerp.usp.br
Recebido: 17 de fevereiro de 2011 Aprovado: 11 de novembro de 2011 